\title{
Missing the boat: fatal ending to a missed case of Charcot arthropathy
}

\author{
Anna Luiza Galeazzi Rech1, Yvon Stüve1, Andreas Toepfer² and Katrin E Schimke³ \\ ${ }^{1}$ Kantonsspital Sankt Gallen, Klinik für Allgemeine Innere Medizin/Hausarztmedizin, Sankt Gallen, Switzerland, \\ ${ }^{2}$ Kantonsspital Sankt Gallen, Klinik für Orthopädische Chirurgie und Traumatologie des Bewegungsapparts, Sankt \\ Gallen, Switzerland and ${ }^{3}$ Kanatonsspital Sankt Gallen, Klinik für Endokrinologie, Diabetologie, Osteologie und \\ Stoffwechselerkrankungen, Sankt Gallen, Switzerland
}

Correspondence should be addressed to K E Schimke Email

katrin.schimke@hin.ch

\section{Summary}

Acute Charcot neuropathic osteoarthropathy (CN) is a clinical entity which can easily go unrecognized in its acute early stages due to lack of awareness and unspecific presentation. However, missing early diagnosis can lead to severe complications. We present the case of a 72-year-old male patient who went through the natural course of the disease unnoticed before the very eyes of his physicians leading to a tragic end. We aim to raise awareness for this rare diabetic complication, emphasizing the necessity of early diagnosis and adequate, interdisciplinary treatment.

\section{Learning points:}

- Clinical signs and symptoms of acute Charcot neuropathic osteoarthropathy (CN).

- Red flags.

- Importance of early diagnosis and correct treatment.

- Diagnostic challenges of acute CN.

- Awareness of high morbidity and mortality.

\section{Background}

Charcot neuropathic osteoarthropathy $(\mathrm{CN})$ is a poorly known, often misdiagnosed clinical entity with a great variety of clinical presentations. The condition is named after the French neurologist Jean-Martin Charcot, who first described progressive arthropathic joint changes in patients with tabes dorsalis (1). Although diabetes mellitus complicated by neuropathy is the most common cause of a $\mathrm{CN}$ in the western world today, it is also seen in other conditions complicated by neuropathy such as idiopathic neuropathy, alcoholism and rheumatoid arthritis (2).

The pathogenesis is still not fully understood, puzzling even experienced specialists. The interaction of several component factors (diabetes, sensory-motor neuropathy, autonomic neuropathy, trauma and metabolic abnormalities of bone) results in an acute localized inflammatory condition leading to varying degrees and patterns of progressive bone destruction, subluxation, dislocation and deformity in the final, chronic state. The current belief is that once the disease is triggered in a susceptible individual, it is mediated through a process of uncontrolled inflammation in the foot (2). Two wellaccepted theories, not necessarily excluding each other, are discussed (3):

The neurotraumatic theory assumes, that often minor, unrecognized acute, subacute or cumulativerepetitive trauma, occasionally iatrogenic (e.g. surgery), but also infectious (e.g. osteomyelitis), causes some tissue or bone damage resulting in a physiologic inflammatory reaction secondary to the release of various proinflammatory cytokines (e.g. tumor necrosis factor (TNF) alpha, interleukin (IL) -1beta and IL-6). Reduced nociception secondary to sensory neuropathy induces 
affected individuals to not rest appropriately. Thereby the inflammatory response is maintained and even exaggerated, opposing anti-anflammatory cytokines like IL-4, IL-10 as well as osteoprotegerin. Mainly TNFalpha upregulates the receptor activator of nuclear factor $K$ (RANK) and its ligand (RANKL) system, which is responsible for abnormally intense osteoclastogenesis and excessive bone turnover, impairing proper bone remodelling, eventually leading to progressive bone destruction.

The second theory hypothesizes a neurovascular cause. Impairment of the physiologic vasomotoric reflex secondary to autonomic neuropathy results in a state of hyperaemia. The increased blood flow leads to a capillary leak, increasing the compartmental pressure and therefore favoring deep tissue ischemia. This could result in trophic damage to ligaments and tendons resulting in joint instability. The hyperaemia might also lead to increased delivery of monocytes and osteoclasts resulting in additional osteoclastic activity, further weakening the bone.

However, it seems safe to say, that sensory neuropathy with subsequently impaired nociception is a consistent feature of the affected limbs and local blood supply is usually sufficient to mount an inflammatory response (4).

Diagnosis is based on patient's history, clinical examination and imaging. Patients typically present with a markedly swollen, warm and often erythematous foot. The temperature difference to the contralateral foot varies from at least $2^{\circ} \mathrm{C}$ up to several degrees(5). A prerequisite finding is some degree of peripheral sensory neuropathy. Pain might be felt, but the level of discomfort related to the degree of injury is usually clearly diminished if compared to healthy individuals (3). Although the clinical signs of inflammation are often impressive, inflammatory markers are usually not elevated significantly. Plain $\mathrm{x}$-rays are recommended as the first diagnostic imaging modality until today, providing information on bone structure, alignment and mineralization. However, early in the course of disease, before the development of gross musculoskeletal pathology, they might be normal. Therefore, cellulitis, deep vein thrombosis or gout are common misdiagnoses. MRI is the diagnostic tool of choice in this stage, demasking typical subtle early changes like subchondral bone marrow oedema with or without microfracture (6). Any part of the foot might be affected, resulting in five anatomic regions defined by Sanders and Frykberg (7). Therefore, in the absence of a foot lesion, a relatively painless, overheated, red and swollen foot in a patient with neuropathy should be considered as $\mathrm{CN}$ unless proven otherwise (8).

The greatest diagnostic challenge arises in an already ulcerated foot with respect to differentiating acute neuropathic from infectious changes (diabetic foot osteomyelitis (DFO), septic arthritis), especially since both may be present at the same time. In this case ulcer location, positive probing to bone or markedly elevated inflammatory markers, especially a high erythrocyte sedimentation rate (ESR), may help (9). MRI is reported to have a sensitivity of 0.9 and specificity of 0.8 in diagnosing DFO. However, differentiating DFO from reactive bone marrow oedema and $\mathrm{CN}$ remains challenging as every diagnostic imaging modality has strengths and limitations. There is some evidence that newer modalities (e.g. dynamic contrast-enhanced MRI) might offer advantages and other techniques (e.g. ${ }^{18} \mathrm{~F}$-fluorodeoyglucose (FDG)-PET/CT and ${ }^{99 m} \mathrm{Tc}-$ exametazime (HMPAO)-labeled leukocyte scintigraphy) might have higher specificity than MRI under special circumstances. They certainly present diagnostic alternatives when MRI is contraindicated (8).

Aside from early diagnosis, instant, optimal treatment of $\mathrm{CN}$ is crucial in order to prevent major deformity, resulting in unphysiological pressure points, foot ulcers and other complications, often making major amputation inevitable. The mainstay of treating acute $\mathrm{CN}$ is offloading, ideally in an irremovable total contact cast. (2). The cast should be changed frequently to avoid 'pistoning' due to subsiding oedema and ensure intact skin conditions. Duration is mainly guided by clinical assessment and ranges from weeks to months (10). The advantages of strict offloading need to be balanced against possible undesired consequences like increased instability, consecutive risk of falls and deconditioning. Until today there are no pharmacologic remedies to treat the condition (10). Clinical trial results of anti-resorptive and anabolic agents have to be interpreted with caution for various reasons, therefore prohibiting their use in routine clinical practice. Surgery is usually avoided during the early, inflammatory stage of disease due to fear of poor healing or mechanical failure of fixation. Interventions during the chronic, inactive stage are targeted at inner offloading, for example, by resecting bony prominences or more complex reconstructive procedures, if conservative measures of treatment fail (2). Once the inflammatory process of the acute phase has subsided, transition from cast to footwear needs to proceed cautiously under close surveillance for possible signs of relapse. Individualized 
protective weight bearing through prescription shoes or custom-made orthoses is mandatory to prevent recurrence and ulcers (2). To ensure treatment adherence, patients must understand the disease and its potentially cyclical nature. Careful and repeated instruction in recognizing the signs of their limb-threatening condition and seeking specialized care immediately in this case is peremptory (11).

\section{Case presentation}

We present the case of a 72-year-old male Caucasian with a long history of type- 2 diabetes and the full picture of diabetic micro- and macro-vascular complications.

In July 2018, he presented with a 3-day history of a painful, red, swollen and hyperthermic left lower leg. As the symptoms did not improve on Co-Amoxicillin p.o. commenced by his GP, he was hospitalized at a regional general hospital. On admission the patient was hemodynamically stable. The left foot and lower leg were badly swollen and tender. Interdigital spaces showed macerated skin with fissures, possible entrance portals for soft tissue infection. Foot pulses were not palpable. The ankle brachial index (ABI) was 0.8 bilaterally, suggesting a mild arterial perfusion deficit. Pallanaesthesia as well as reduced monofilament sensation were consistent with significant impairment of nociception. Ear-temperature was $37.6^{\circ} \mathrm{C}$, BP $161 / 86 \mathrm{mmHg}$, pulse $96 / \mathrm{min}$. Blood tests showed high inflammatory markers: CRP $182 \mathrm{mg} / \mathrm{L}$ (norm <5), leukocytes $15.2 \mathrm{~g} / \mathrm{L}$ (norm <10.0) with $86 \%$ neutrophils, eGFR $80 \mathrm{~mL} / \mathrm{min} / 1.73 \mathrm{~m}^{2}$, HbA1c $90 \mathrm{mmol} /$ mol (norm 22-39).
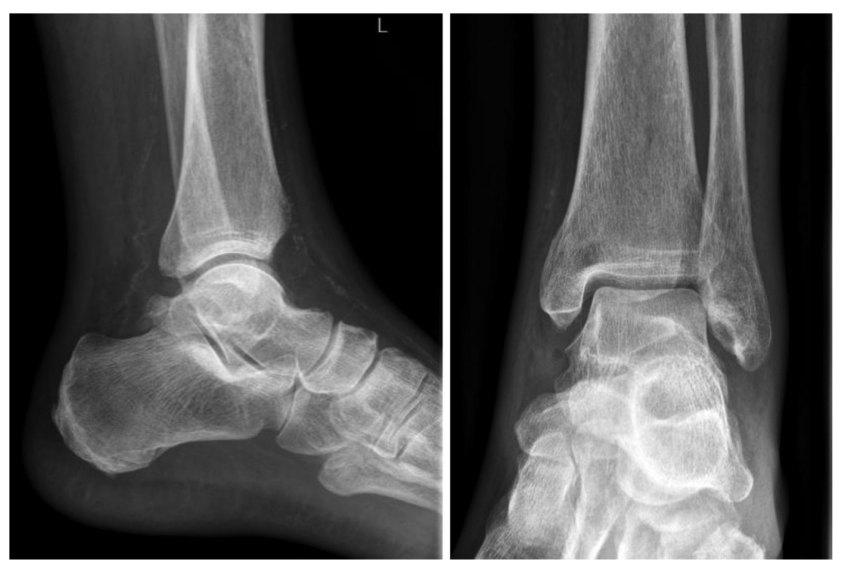

Figure 1

Conventional x-ray of the left ankle at initial presentation in July 2018.

\section{Investigation}

X-ray showed normal osseous structures, but minimal effusion in the left upper ankle joint (Fig. 1). MRI showed unspecific bone marrow oedema of the ankle joint, tarsus and metatarsus, not typical for infection. Due to slow clinical improvement a skin biopsy was performed, resulting in the histological diagnosis of stasis dermatitis, rather than cellulitis.

\section{Treatment}

Antibiotic therapy was initially changed to Amoxicillin/ clavulanic acid $3 \times 2.2 \mathrm{~g}$ i.v. and then to Piperacillin/ Tazobactam i.v. as clinical signs and symptoms did not improve. After a hesitant course, the patient was finally discharged.

\section{Outcome and follow-up}

In January 2019, the patient presented to a different regional hospital, this time complaining of acute pain in both distal legs. On the left the pain was described as electrifying, originating from the medial malleolus. Clinically, significant bilateral lower extremity oedema was found with inflammatory signs restricted to the left lower leg. The patient was afebrile and hemodynamically stable. This time blood tests showed only a mildly elevated CRP of $28 \mathrm{mg} / \mathrm{L}$ and leucocytes within normal limits. Despite of this, cellulitis of the left lower extremity was diagnosed, and i.v. antibiotics started again (Co-Amoxicillin). Meanwhile, a painless second-degree ulcer had developed lateral of the right fifth metatarso-phalangeal (MTP) joint without clinical signs of infection. Clinical examination, pulmonary x-ray and an elevated BNP of $1341 \mathrm{ng} / \mathrm{L}$ (norm $<37$ ) argued for concomitant cardiac decompensation, which was treated with diuretics. X-ray and MRI ruled out osteomyelitis of the right foot. No diagnostic imaging of the left foot was performed at this time.

Because of visibly calcified pedal arteries (mediasclerosis) on $\mathrm{x}$-ray, possibly leading to falsely elevated pressures, a more comprehensive vascular review was ordered. Indeed, this showed markedly reduced toe pressures and pathologic pulse wave forms. Significantly compromised arterial blood flow was confirmed by subsequent bilateral angiography and followed by uncomplicated, successful percutaneous transluminal angioplasty on both sides. After cardiac recompensation and clinical improvement on both lower extremities along with a reduction of the swelling, the patient was discharged after 14 days and 
referred to the diabetic foot clinic at the regional tertiary hospital for further treatment.

Despite adequate wound management and offloading the lesion lateral to the MTP fifth joint on the right did not show adequate progress. However, the patient was not disturbed by this at all. He was complaining about severe pain in his left foot, strongly affecting his mobility. Other than mild erythema, moderate swelling and hyperthermia, no obvious pathology was detected by the treating physicians. Focussing on the new foot ulcer on the right, the urgency of the complaint on the left was unfortunately misjudged: over the next few weeks a gradually progressive deformity of the left ankle joint was observed culminating in an ulcerous lesion with purulent drainage over the left lateral malleolus through which a large wound cavity could be probed straight to the bone (Fig. 2). After all, an x-ray confirmed what was already clinically evident: right before our eyes the complete picture of $\mathrm{CN}$ had developed and progressed unhindered until complete destruction and dislocation of the upper ankle joint (Fig. 3). The ulcer had developed based on the severe deformity with the fibula poking through the skin from inside, opening the way for superinfection. Vital signs showed an auricular temperature of $37.8^{\circ} \mathrm{C}$, BP $111 / 59 \mathrm{mmHg}$, and a pulse rate of $74 / \mathrm{min}$. Inflammatory markers were only mildly raised (CRP $68 \mathrm{mg} / \mathrm{L}$ (norm $<5$ ), leukocytes $6.9 \mathrm{G} / 1$ (norm <10.0) with $81.5 \%$ neutrophils), but renal function had declined to an eGFR of $40 \mathrm{~mL} /$ $\min / 1.73 \mathrm{~m}^{2}$. The patient was hospitalized immediately, assigned to complete bed rest and started on antibiotic therapy with Co-Amoxicillin $4 \times 2.2$ g i.v.. When blood cultures taken prior to antibiotic therapy showed staphylococcus aureus bacteraemia, the antibiotic
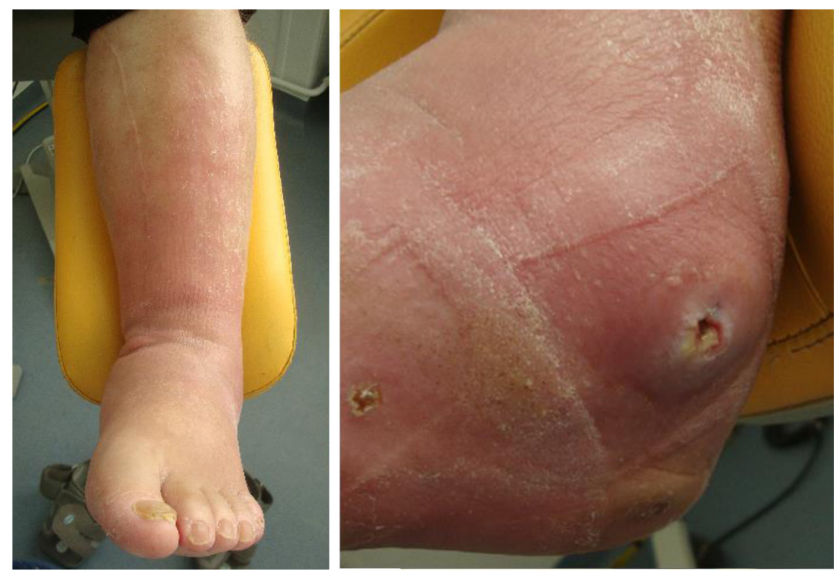

Figure 2

Clinical aspect of the left lower extremity and lateral malleolus in March 2019.

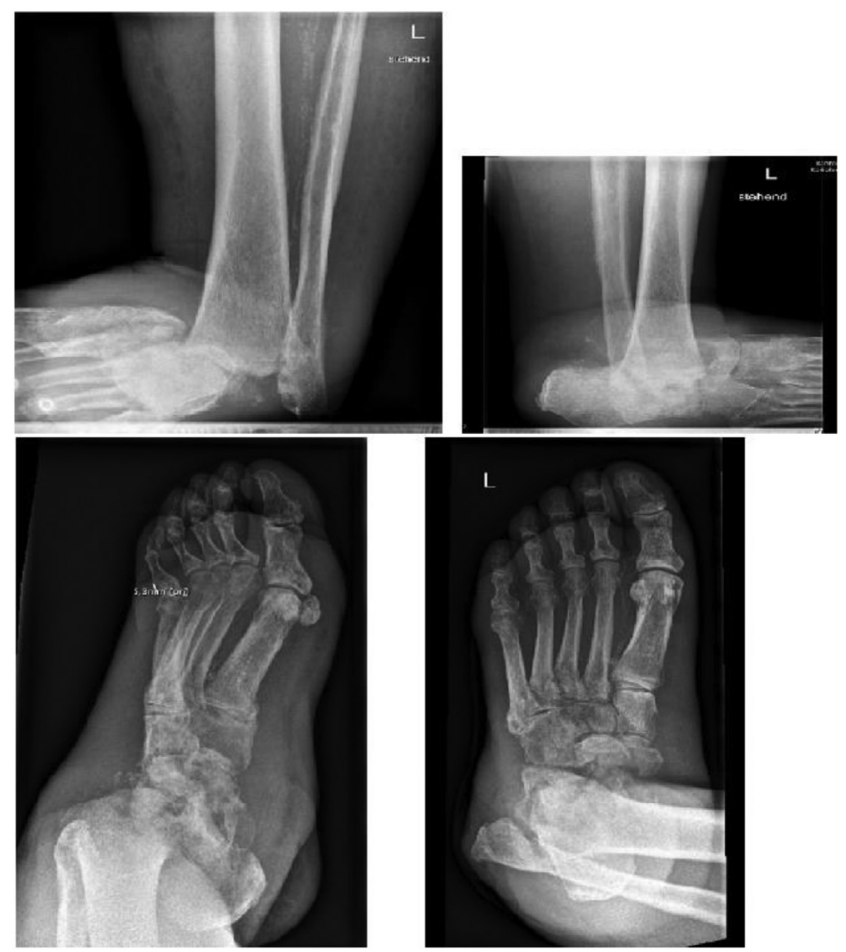

Figure 3

Conventional x-ray of the left foot and ankle in March 2019.

regimen was changed to Cefazolin $2 \times 2 \mathrm{~g}$ i.v.. Parallel to this the patient developed acute kidney injury three with hemodynamic dysfunction, interpreted as acute toxic nephritis secondary to Co-Amoxicillin. With respect to the severe osseous destruction and superinfection, below knee amputation was the only reasonable treatment option at this point, but vehemently rejected by the patient initially. Only two weeks later he and his family finally agreed. Surgery was uneventful and was carried out according to orthopaedic surgical standards. However, six days later multiorgan dysfunction syndrome developed and despite intensive care efforts the patient died on April 11th, 2019.

\section{Discussion}

The tragic course of the case presented here illustrates the possible consequences of misinterpreting clinical and diagnostic findings by medical professionals unaware of a rare, but very important differential diagnosis of a red, hot, swollen, neuropathic foot without ulceration.

Signs of possible incipient $\mathrm{CN}$ were clearly present from the beginning: while the $\mathrm{x}$-ray, then, did not show any osseous pathology, MRI showed diffuse bone marrow oedema of the ankle joint, tarsus and metatarsus, not typical for infection, but consistent with possible 
early $\mathrm{CN}$. In the absence of a full thickness foot lesion, but high inflammatory markers, the treating physicians assumed cellulitis and started antibiotic treatment and neglected the subtle magnetic resonance tomographic signs. However, no later than at the second presentation in 2019 with similar signs and symptoms on the left lower extremity, but relatively low inflammatory markers this time, could have rang a bell to consider acute $\mathrm{CN}$ as a possible differential diagnosis and proceed with further investigations. Whether it had been there from the beginning or had been triggered by preceding cellulitis, remains speculative. Unfortunately, no diagnostic imaging was performed of the left foot and ankle at this moment as the treating physicians were distracted focussing on the newly developed ulcer on the opposite foot and other clinical problems like heart failure.

Presumably, the unhindered natural course of the disease as observed in this case, could have been prevented, if the early signs had been interpreted correctly, proper investigations had been ordered and adequate treatment with strict offloading been initiated. We believe however, that the deleterious, fulminant destructive neuro-osteopathic process witnessed after the second hospitalization, might actually have been triggered by the revascularization procedure. Optimization of blood supply has been described as a possible risk factor for the development of neuropathic osteoarthropathy (12).

The fact, that the patient complained about severe pain might be considered atypical and certainly misguided some of the physicians involved. Some pain is felt by about $50 \%$ of the affected patients (13). Nevertheless, considering that the patient walked into the outpatient clinic on a completely dislocated ankle joint (Figs 2 and 3), clearly illustrates that his symptoms were not correlating to the clinical findings at all. The tragic course of the presented case underlines the difficulty of the diagnosis, especially for professionals with little to no experience in the area of $\mathrm{CN}$ as well as the deleterious consequences of missing the diagnosis. Even if diagnosed earlier, the outcome of our patient would have been uncertain as $\mathrm{CN}$ of the ankle joint is the most difficult location to treat, usually demanding early complex surgical interventions and even longer periods of off-loading (2). The outcome of this elderly man also underlines the fact that patients affected usually suffer from a lot of comorbidities and have a high mortality (14).

In sharing this fatal case with a broader audience, we aim to raise awareness of this rare condition, helping to speed up correct diagnosis and initialize adequate treatment.

\section{Declaration of interest}

The authors declare that there is no conflict of interest that could be perceived as prejudicing the impartiality of the research reported.

\section{Funding}

This research did not receive any specific grant from any funding agency in the public, commercial or not-for-profit sector.

Patient consent

Written informed consent was obtained from the patient's family.

Author contribution statement

A L Galeazzi and Y Stüve wrote the draft, A Toepfer and K E Schimke revised it.

\section{References}

1 Ramanujam CL \& Zgonis T. The diabetic Charcot foot from 1936 to 2016: eighty years later and still growing. Clinics in Podiatric Medicine and Surgery 201734 1-8. (https://doi.org/10.1016/j. cpm.2016.07.001)

2 Rogers LC, Frykberg RG, Armstrong DG, Boulton AJ, Edmonds M, Van GH, Hartemann A, Game F, Jeffcoate W, Jirkovska A, et al. The Charcot foot in diabetes. Diabetes Care 201134 2123-2129. (https:// doi.org/10.2337/dc11-0844)

3 Hochlenert D, Engels G, Morbach S, Schliwa S \& Game FL. Diabetic Foot Syndrome, S 336. Springer Verlag, 2018.

4 Strotman PK, Reif TJ \& Pinzur MS. Charcot arthropathy of the foot and ankle. Foot and Ankle International 201637 1255-1263. (https:// doi.org/10.1177/1071100716674434)

5 van Netten JJ, Prijs M, van Baal JG, Liu C, van der Heijden F \& Bus SA. Diagnostic values for skin temperature assessment to detect diabetes-related foot complications. Diabetes Technology and Therapeutics 201416 714-721. (https://doi.org/10.1089/ dia.2014.0052)

6 Kiuru MJ, Pihlajamaki HK, Hietanen HJ \& Ahovuo JA. MR imaging, bone scintigraphy, and radiography in bone stress injuries of the pelvis and the lower extremity. Acta Radiologica 200243 207-212. (https://doi.org/10.1080/028418502127347790)

7 Frykberg RG, Zgonis T, Armstrong DG, Driver VR, Giurini JM, Kravitz SR, Landsman AS, Lavery LA, Christopher Moore J, Schuberth JM, et al. Diabetic foot disorders: a practical guideline. Journal of Foot and Ankle Surgery 200645 1-66.

8 Uccioli L. Why is Charcot foot commonly misdiagnosed? Diabetes Management 20122 81-84. (https://doi.org/10.2217/dmt.12.6)

9 Lipsky BA, Senneville É, Abbas ZG, Aragón-Sánchez J, Diggle M, Embil JM, Kono S, Lavery LA, Malone M, van Asten SA, et al. Guidelines on the diagnosis and treatment of foot infection in persons with diabetes (IWGDF 2019 update). Diabetes/Metabolism Research and Reviews 202036 (Supplement 1) e3280. (https://doi. org/10.1002/dmrr.3280)

10 Petrova NL \& Edmonds ME. Conservative and pharmacologic treatments for the diabetic Charcot foot. Clinics in Podiatric 
Endocrinology

Diabetes \& Metabolism

CASE REPORTS

\section{A L Galeazzi Rech and} others
Charcot arthropathy with fatal ending
ID: 20-0013; June 2020

DOI: 10.1530/EDM-20-0013
Medicine and Surgery 201734 15-24. (https://doi.org/10.1016/j. cpm.2016.07.003)

11 Milne TE, Rogers JR, Kinnear EM, Martin HV, Lazzarini PA, Quinton TR \& Boyle FM. Developing an evidence based clinical pathway for the assessment, diagnosis and management of acute charcot neuro-arthropathy: a systematic review. Journal of Foot and Ankle Research 20136 30. (https://doi.org/10.1186/1757-1146-6-30)

12 Edelmann SV, Kosofsky EM, Paul RA \& Kozak GP. Neuroosteoarthropathy (Charcot's joint) in diabetes mellitus following revascularization surgery. Three case reports and a review of the literature. Archives of Internal Medicine 1987147 1504-1508. (https:// doi.org/10.1001/archinte.147.8.1504)

13 Palena LM, Brocco E, Ninkovic S, Volpe A \& Manzi M. Ischemic Charcot foot: different disease with different treatment? Journal of Cardiovascular Surgery $2013 \mathbf{5 4}$ 561-566.

14 Armstrong DG, Swerdlow MA, Armstrong AA, Conte MS, Padula WV $\&$ Bus SA. Five year mortality and direct costs of care for people with diabetic foot complications are comparable to cancer. Journal of Foot and Ankle Research 202013 16. (https://doi.org/10.1186/s13047-02000383-2)

Received in final form 7 May 2020

Accepted 26 May 2020 